\title{
LITERATURE REVIEW OF ANEURYSMAL SUBARACHNOID HEMORRHAGE FROM INCIDENCE TO TREATMENT OPTIONS
}

\author{
Ieva Buce-Satoba*,**,***, Daina Rozkalne ***, Jevgenijs Stepanovs *, Biruta Mamaja*,****, Gaida \\ Krumina $* * * *, * * * * *$, Agnese Ozolina*,**** \\ * Riga East University Hospital, Clinic of Anesthesiology, Riga, Latvia \\ ** Riga Stradins University, Doctoral studies of Medicine, Riga, Latvia \\ *** Riga East University Hospital, Intensive Care Clinic, Riga, Latvia \\ **** Riga Stradins University, Department of Anesthesiology and Reanimatology, Riga, Latvia \\ $* * * * *$ Riga East University Hospital, Department of Radiology, Riga, Latvia \\ ****** Riga Stradins University, Department of Radiology, Riga, Latvia
}

\begin{abstract}
Summary
Introduction. Aneurysmal subarachnoid haemorrhage (SAH) is associated with high mortality and morbidity. Rebleeding, cerebral vasospasm (VS) with delayed cerebral ischemia (DCI) are major complications after SAH associated with poor neurological outcome.
\end{abstract}

Aim of the study. To summarize the existing research data on the SAH from incidence, risk factors and clinical presentation to diagnostic, monitoring and treatment options after SAH.

Materials and Methods. Literature review was carried out to identify factors associated with SAH using specific keywords (aneurysmal subarachnoid haemorrhage, rebleeding, cerebral vasospasm, delayed cerebral ischemia) in the PUBMED database. In the time period from 2000 to 2019, 34 full articles were reviewed.

Results. According to the literature, the key risk factors for cerebral aneurysms and the SAH are hypertension, smoking, chronic alcohol abuse, family history of intracranial aneurysms in first-degree relatives and female sex. The key risk factor for early complication - rebleeding after SAH - is hypertension. The factors responsible for late complications - cerebral VS and DCI after SAH - are initially lower Glasgow coma scale and higher grades of Fisher scale, where grade IV and III predict cerebral VS in 31-37\%. Furthermore, hyperglycaemic state, hyponatremia, hypotension and cerebral hypoperfusion, increased level of Troponin correlate with the incidence of cerebral VS and DCI. Although the golden standard to detect cerebral VS is digital subtraction angiography, CT angiography has become a routine examination. Transcranial doppler sonography is recommended and regional cerebral oximetry also seems to be promising. To avoid rebleeding for wide-necked, gigantic aneurysms or when $\mathrm{SAH}$ is combined with intraparenchymal hematoma, surgical clipping is preferred. For posterior circulation aneurisms, poor grade $\mathrm{SAH}$ and patients with age $>70$ years superior is endovascular treatment. To avoid late complications, the pharmacological method is used with Nimodipine.

Conclusions. SAH is still associated with poor clinical outcome due to the development of early and late complications. The highest risk patients are those with low Glasgow coma scale and high grades of Fisher scale. Timely performed obliteration methods of the ruptured aneurysm are crucial and Nimodipine is the main agent to prevent cerebral VS and DCI.

Keywords: aneurysmal subarachnoid haemorrhage, rebleeding, cerebral vasospasm, delayed cerebral ischemia.

\section{INTRODUCTION}

Subarachnoid haemorrhage (SAH) from ruptured intracranial aneurysms is a subtype of stroke with high fatality and morbidity. Mortality is commonly caused by neurological injury resulting from the initial bleeding and rebleeding, and from cerebral VS with delayed cerebral ischemia (DCI).

Epidemiology and risk factors for aneurysm formation and rupture

Epidemiology of the aneurysm formation and rupture. $\mathrm{SAH}$ accounts for $5-10 \%$ of all strokes and half of patients with SAH are younger than 55 years and most survivors have long-term disability or cognitive impairment due to DCI.
In a meta-analysis including 75 studies and 8176 patients, the worldwide incidence of SAH was recorded to be decreased by $1.7 \%$ from 10 per 100000 personyears in 1980 to 6 in 2010. The incidence of SAH in Europe was 6 per 100000 person-years in 2010 (1). Moreover, the incidence of aneurysmal SAH varies by geographic region from 4 per 100000 person-years in Asia excluding Japan to 7 per 100000 person-years in Australia and New Zealand and even 28 to 100000 person-years in Japan in 2010 (1).

In Latvia the incidence of SAH in the period of the last 5 years was from 9 to 11 per 100000 population according to the data from the Centre for Disease Prevention and Control of Latvia. 
Most of all cerebral aneurysms are located in the anterior circulation of the brain - the internal carotid artery, the anterior and middle cerebral arteries, and their branches. Only 10-20\% of cerebral aneurysms are located in the posterior circulation of the brain - the vertebral, basilar and posterior cerebral arteries, and their branches (4). The circle of Willis demonstrating anterior and posterior cerebral circulation is depicted in Fig. $1 \mathrm{~A}$ and $1 \mathrm{~B}$ below.

Risk factors of the aneurysm formation and rupture. The important risk factors contributing to the development of cerebral aneurysms are hypertension, smoking, chronic alcohol abuse, family history of intracranial aneurysms in first-degree relatives and female sex (2). The age-specific and sex-specific SAH incidence for $2133 \mathrm{SAH}$ patients were reported by Etminan and co-authors (1). The incidence of $\mathrm{SAH}$ increases in women older than 55 years in the menopausal period. The overall sex-specific incidence of SAH was 12 (95\% CI, 9.5-13.9) per 100000 personyears in women vs $9(95 \%$ CI, 7.7-11.3) in men. There is a connection between two risk factors - age and female sex - and uncontrolled blood pressure (BP) levels and smoking habits. With every millimetre of mercury decrease in systolic BP, the overall ageadjusted and sex-adjusted incidence of SAH declined by $7.1 \%(95 \%$ CI, 5.8-8.4) and decrease in diastolic BP by $11.5 \%$ (95\% CI, 8.8-14.3). With every percentage decrease in smoking prevalence, the overall ageadjusted and sex-adjusted incidence of SAH decreased by $2.4 \%$ (95\% CI, 1.6-3.3) (1).

Excessive alcohol intake is considered to damage the endothelium and has also been associated with elevated blood pressure. The risk of SAH grows with increasing amounts of alcohol (13).

The first-degree relatives of patients with aneurysmal SAH have a 4 to 7 -fold increased risk compared with the general population (12). Autosomal dominant polycystic kidney disease is strongly associated with intracranial aneurysms and has a 2 to 4 times higher prevalence rate of SAH (2).

In contrast, in a meta-analysis including 4327 patients with SAH decreased association between diabetes mellitus and SAH risk was reported. These results might be explained by a change in their lifestyles and well-controlled arterial hypertension. Moreover, the diabetes mellitus patients are likely to have higher mortality caused by diseases other than SAH $(13,14)$. Sympathomimetic drug such as appetite suppressant phenylpropanolamine, methamphetamine and cocaine also had been associated with aneurysmal $\mathrm{SAH}$. Cocaine can induce cerebral VS that can lead to DCI and even worse outcome $(13,15)$.

\section{Clinical presentation of SAH}

Neurological presentation. Most aneurysms remain undetected until rupture and in only $5 \%$ of cases they can be manifested by a variety of neurological symptoms - epileptic seizures, mass effect from aneurysmatical sac, thromboembolic events and others (4). Depending on localization, SAH may be exposed in several ways. Severe headache, bilateral temporal hemianopia and bilateral lower extremity weakness, unilateral nerve palsy, facial or orbital pain, epistaxis, progressive vision loss and/or ophthalmoplegia and symptoms of brainstem dysfunction are various symptoms after SAH (2). Often the notable symptom is sudden, severe headache, often described as the worst headache in one's life (6). Moreover, loss of consciousness or seizures may be the first manifestation of SAH (16). Additionally, for an acute SAH meningeal symptoms, photophobia, nausea and vomiting are typical symptoms, but up to $50 \%$ of $\mathrm{SAH}$ patients may have a normal neurological exam $(4,6)$.

Non-neurological presentation. In all SAH patients massive sympathetic nervous system activation occurs leading to an increase of circulatory catecholamine. That seems to be the reason of genesis of some systemic complications after initial ictus. Catecholamineinduced stress through production of toxic cytokines may induce different organ dysfunction, including myocardial cytolysis, high-pressure pulmonary edema, stress hyperglycaemia, electrolyte imbalance and leucocytosis (17).

Cardiac changes primary reflects the severity of neurological injury and are reversible in most of cases (2). Neurocardiogenic injury following SAH includes electrocardiographic (ECG) abnormalities, arrhythmias, myocardial infarction, left ventricular dysfunction, elevation of Troponin levels and even cardiac arrest (17). More than $90 \%$ of patients with acute SAH have ECG abnormalities (4), including ST and $\mathrm{T}$ wave changes, QT prolongation and $\mathrm{U}$ waves. Stimulation of hypothalamus may induce ECG abnormalities without associated myocardial injury (2, 17). The ischemic neuronal degeneration in the nodose ganglia leads to supraventricular and ventricular heart rhythm disturbances. Heart rate variability is a potential marker of reversible cardiac injury and connection between QT prolongation, tachycardia and development of angiographic cerebral VS has been detected $(2,17)$.

Approximately $10 \%$ of all SAH patients have left ventricle systolic dysfunction. Due to lack of estradiol postmenopausal women after poor-grade SAH are predisposed to develop heart wall motion abnormalities. Impaired left ventricle function may lead to cardiovascular instability, pulmonary edema and DCI as a result from hypotension and cerebral hypoperfusion $(4,17)$.

Elevated serum levels of Troponin are presented in $17-28 \%$ of the SAH patients. The elevation degree correlates with an increased risk of cardiovascular complications, cerebral VS induced DCI and poor neurological outcome. Overall, higher level of Troponin is associated with increased mortality rates $(2,17)$. Sympathetic activation after SAH increases BP leading to hypertension. A recent large randomized controlled trial including 2794 patients shows that systolic BP can 
be safely reduced to $140 \mathrm{mmHg}$ to decrease the risk for rebleeding without increasing the risk for ischemic brain complications (2).

The most common pulmonary complications after $\mathrm{SAH}$ are pneumonia, aspiration and neurogenic pulmonary edema (NPE). These are the most frequent extracerebral causes of death. Hypoxia is found in $43 \%$ to $92 \%$ of cases in the acute stage of SAH and most often is due to NPE, which usually develops within the first week from the ictus with a peak around 3 day. Cardiac dysfunction and elevated BP are responsible for NPE in early phase of SAH, and hypervolemia and low cardiac contractility 7 days after (17). NPE reflects the severity of the subarachnoid bleed and is associated with poor outcome (2).

Stress hyperglycaemic is seen in $70 \%$ to $90 \%$ of acute $\mathrm{SAH}$ patients due to the activation of the hepatic and pancreatic sympathetic nerve fibres and catecholamine induced development of hepatic insulin resistance. Hyperglycaemia exacerbates $\mathrm{SAH}$-induced brain injury by enhancing the mitochondrial dynamic imbalance, apoptosis and inflammation. The glucose level at admission is related to the severity of initial haemorrhage and is an independent predictor of the occurrence of DCI and poor outcome. Insulin therapy improves the prognosis for patients with SAH (17).

Finally, electrolyte disturbances are found in approximately $30 \%$ of all SAH patients. Hypokalaemia is detected most often due to catecholamine overload. In cases of severe hypokalaemia, potassium should be supplemented (17). Hyponatremia occurs less often approximately in $10-35 \%$ of patients. All patients have increased urine output and urinary excretion of sodium due to cerebral salt-wasting syndrome, syndrome of inappropriate $\mathrm{ADH}$ secretion or secretion of adrenomedullin. Adrenomedullin is a vasorelaxant peptide, which is secreted into the cerebrospinal fluid (CSF) from the choroid plexus and exert natriuretic effects in the kidney. Adrenomedullin concentration in CSF is higher during the late period of SAH and correlates with hyponatremia and DCI. The aim of the treatment of hyponatremia is to maintain a positive salt balance and water replacement $(4,17)$ to improve prognosis.

\section{Assessment of SAH}

Clinical assessment. The severity of SAH clinically is assessed and graded using different scales. The clinical condition at admission is an important factor to measure the severity of initial neurological injury, predict functional outcome and to guide treatment. The Hunt and Hess Scale, the World Federation of Neurological Surgeons Scale and the Glasgow Coma Scale (GCS) are the most popular scales. The Hunt and Hess Scale is a 5-category grading scale that classifies patients with SAH according to their clinical condition and is used to predict prognosis and outcome. Higher grades in the Hunt and Hess Scale are associated with worse outcomes as depicted in Table 1. Because of some limitations in the Hunt and Hess Scale, the World
Federation of Neurological Surgeons Scale was created. This scale is based on the GCS, but with focal deficits. The follow up neurological status is assessed using the GCS, which has a prognostic value and less observer variability $(7,18)$.

Radiological assessment. The Fisher scale is a radiological classification, which represents the size of SAH on a cranial computer tomography (CT) scan and is useful in predicting the occurrence and severity of cerebral VS (Table 2), DCI, and patient's overall outcome (5).

Based on a reasonable suspicion of SAH, non-contrast CT is the first diagnostic tool. This helps to confirm the diagnosis of SAH and exclude other pathologies such as intracranial haemorrhage, malignancy or abscess. If non-contrast CT is performed within 6 hours of the symptom onset, the sensitivity is near $100 \%$. If the $\mathrm{CT}$ is negative within of headache onset, a risk of an acute $\mathrm{SAH}$ is less than $1 \%$. A lumbar puncture may be considered when non-contrast CT is not definitive but clinical suspicion remains $(4,6,19)$.

Although, the gold standard for detection of cerebral aneurysms is digital subtraction angiography, meta-analysis of 4097 patients proved that the CT angiography is an accurate tool to diagnose ruptured intracranial aneurysm. The chance of missing a ruptured aneurysm at the CT angiography is no more than $2 \%$ if they are small and located near the skull base. Nowadays, the CT angiography has become a routine examination fully integrated into the imaging and treatment algorithms for patients with SAH in many centres in Europe (5).

Magnetic resonance imaging (MRI) is not used routinely for diagnostic purposes of acute SAH. This method may be useful in certain atypical cases and with a long delay from ictus $(4,6)$.

\section{Complications of SAH}

SAH complications can be divided into early, late and chronic as depicted in Table 3 (10).

Early complications. Rebleeding increases mortality by $70 \%$. The highest risk of rebleeding is within the first 6 hours after the ictus. Reported rates of rebleeding within the first 24 hours range from 4-13.6\%. Risk factors are high systolic blood pressure, low Hunt and Hess Scale grades, intracerebral or intraventricular hematomas, posterior circulations aneurysms and a size more than $10 \mathrm{~mm}(10,20)$.

Occurrence of hydrocephalus varies from $6-67 \%$. Main causes are obstruction of the CSF flow by blood products or adhesions, reduction of CSF absorption at the arachnoid granulations or by increase in the CSF secretion. About 20\% hydrocephalus occurs acute in the first 3 days, usually due to the mass effect of blood clots within the ventricles and aqueduct, and subacute in 4-14 days. Hydrocephalus impairs patient's neurologic function and leads to deterioration of functional outcomes. Chronic hydrocephalus develops in $10-20 \%$ of all patients with SAH mostly due to the fibrosis and adhesions of leptomeningeal and arachnoid 
granulations. Higher risk of shunt-dependency can be observed in patients with intraventricular haemorrhage, greater haemorrhage volume according to the Fisher score, high Hunt and Hess Scale score, low score of initial GCS, rebleeding, posterior circulation location of ruptured aneurysm and age $>60$ years $(10$, 21).

Up to $26 \%$ of patients have seizures as a complication of $\mathrm{SAH}$ and are associated with a higher mortality risk. According to a nationwide retrospective cohort analysis, younger patients and males have an increased risk to seizures (22).

Late complications. Angiographic cerebral VS is found in up to $70 \%$ of all SAH patients resulting in DCI around $20-30 \%$ leaving the majority of survivors with motor deficit, cognitive dysfunction and reduced quality of life (23). Cerebral VS is defined as narrowing of the large and medium-sized intracranial arteries that begins around day 3 with a peak around day 7-10. The risk typically resolves 21 day after the bleeding. Cerebral VS is a clinical diagnosis and radiographic studies are used to prove the diagnosis (10). Regular and careful bedside examination remains the simplest and most effective way to detect early ischemia in an awake patient, but it is difficult for those in poor neurological condition or comatose (24).

The most accurate method to confirm the cerebral VS remains digital subtraction angiography, but the CT angiography is often employed as well. The CT angiography is a less invasive and more accessible method of investigation. Studies comparing the CT angiography with digital subtraction angiography have concluded that the CT angiography has a higher sensitivity and specificity in the cerebral VS diagnosis (23). Nevertheless, contrast media containing iodine have direct spastic effect by hyperosmolarity and also by stimulating of vasomotor receptors that facilitate vasoconstriction (25)

The CT perfusion is a non-invasive method, which has gained popularity because of its easy use, speed of image acquisition and good correlation with digital subtraction angiography (24). Also, MRI perfusion, single-photon emission CT, quantitative stable xenonenhanced CT and positron emission tomography can be used to detect early cerebral ischemia, but they are not widely used in practice due to limited diagnostic possibility to detect causative blood vessel (23).

Transcranial Doppler Sonography (TCD) is a noninvasive, ultrasound method of detection of the cerebral VS. It can be easily performed at the bedside, has no serious side effects and is repeatable if a patient has an adequate acoustic window. In many recommendations, the TDC is mentioned as a tool for monitoring and detecting large artery proximal vasospasm but not in peripheral branches. Lack of the TCD is that it is highly operator-dependent $(10,24)$.

Other monitoring methods include continuous electroencephalography, intracranial electroencephalography, brain tissue oxygen monitoring (direct probe cerebral oximetry), cerebral microdialysis, jugular venous bulb oximetry. The main disadvantages of the previously mentioned methods are that most of them are invasive with potential risks and they provide information mainly on cerebral hemodynamic and metabolism (23, 24).

Regional cerebral oximetry (near-infrared spectroscopy) is a non-invasive, simple, bedside, continuous monitoring method of cerebral oxygenation. Near-infrared spectroscopy can measure the regional cerebral haemoglobin saturation on oxygen in an area containing arteries, veins and capillaries with predominantly venous distribution (11). Probes are secured to the skin of forehead and through two photodetectors the near-infrared light penetrates superficial layers and is either absorbed by chromophores (oxy- and deoxyhemoglobin) or scattered within the tissue (24). During the cerebral VS, the decrease in the blood flow results in impaired oxygen delivery leading to reduced oxygen saturation of the venous blood. As regional cerebral oximetry reflects mainly the oxygen saturation in the venous blood, it could indicate arterial spasm and compromise the circulation (11).

In meta-analysis where standard was compared with advanced hemodynamic monitoring (pulse index, continuous cardiac output, pulse densitometry, pulmonary artery catheterization) in relation to the patient management and clinical outcomes after $\mathrm{SAH}$, invasive hemodynamic monitoring helped to reduce the incidence of DCI, but there were not differences in neurological outcome, pulmonary edema onset or fluid intake between the two groups. Further studies are required for monitoring standards in patients after SAH (34).

\section{Treatment of SAH}

The main aim in the early stage of SAH is to prevent rebleeding before microsurgical or endovascular obliteration of aneurysm is obtained $(2,8,10,26)$. Rebleeding is associated with very high mortality (26). Medical treatment to prevent rebleeding. According to the AHA/ASA Guideline, acute hypertension should be controlled after SAH and until aneurysm obliteration with a titratable agent to balance the risk of stroke, hypertension-related rebleeding and maintenance of cerebral perfusion. Systolic blood pressure to $<160 \mathrm{mmHg}$ is reasonable (Class I; Level of Evidence B). In other sources, systolic blood pressure lowering to $140 \mathrm{mmHg}$ is recommended without increasing the risk for ischemic complications. Drugs like labetalol, esmolol, nicardipine, clonidine and other short acting agents are recommended to control blood pressure and reduce hypertension. Sodium nitroprusside, nitro glycerine and hydralazine are not recommended, as they may raise the intracranial pressure $(2,4,6)$. In addition to blood pressure management, potential stress factors like pain, agitation and anxiety must be removed or treated to reduce catecholamine-induced stress and 
end-organ catecholamine-mediated injury $(4,17,27)$. Antifibrinolytic therapy may reduce the incidence of aneurysm rebleeding when there is delay in aneurysm obliteration. Use of short-term ( $<72$ hours) therapy with tranexamic acid or aminocaproic acid is reasonable to reduce the risk of rebleeding (Class IIa; Level of Evidence B) without increasing the risk of DCI $(2,26)$. There is an increased risk of deep venous thrombosis but not pulmonary thrombosis. Reversing anticoagulation (26) should be accomplished as soon as possible. Antiplatelet agents should be reversed with platelet infusion and desmopressin should be considered (6).

Surgical and endovascular methods of treatment. Surgical clipping or endovascular coiling of the ruptured aneurysm should be performed as early as feasible to reduce the rate of rebleeding after SAH (Class I; Level of Evidence B) (26).

A comparison of the two methods is demonstrated in Table 4 below. During surgical treatment the aneurysm is closed off from its parent vessel by placement of a metal clip to occlude its neck. With endovascular treatment aneurysm is occluded by the placement of platinum spirals (coils) inside it (4). The choice of the method should be made by multidisciplinary decision based on the characteristics of the patient and aneurysm (Class I; Level of Evidence C) (26).

\section{Secondary cerebral vasospasm and DCI}

Despite the research that has been carried out, cerebral VS continues to account for high morbidity and mortality in patients with aneurysmal SAH. There are patients with severe large artery spasm who never become symptomatic and others with quite modest spasm who not only develop symptoms but go on to develop infarction. The risk resolves 21 days after the bleed $(10,23,24,26$.) The mechanism of cerebral VS is likely being a multifactorial pathology that include: endothelial damage and formation of microthrombi, smooth muscle contraction from lysis of subarachnoid blood clots and blood degradation products and haemoglobin released into the subarachnoid space, decreased nitric oxide production and release of the potent vasoconstrictor endothelin-1, cortical spreading depolarization, inflammation-mediated oxidative stress and free radical damage to smooth muscle cells and upregulation of apoptosis pathways following $\mathrm{SAH}$ $(10,23)$.

\section{Treatment to prevent cerebral vasospasm}

Pharmacological interventions. Standard treatment to prevent cerebral VS is nimodipine $60 \mathrm{mg}$ every 4 hours administered orally or via nasogastric tube (Class I; Level of Evidence A) and continued for 21 day. If nimodipine causes hypotension, the dose can be divided into $30 \mathrm{mg}$ every 2 hours or reduced to $30 \mathrm{mg}$ every 4 hours $(23,24,26)$.

Magnesium sulphate has been studied and several small trials of aggressive intravenous magnesium administration suggested benefit. A randomized placebo-controlled trial MASH-2 shown that magnesium is not superior to placebo for reduction of poor outcome after SAH (28). While maintaining magnesium within normal limits in patients with $\mathrm{SAH}$ and hypomagnesemia is reasonable, the current evidence does not support routine prophylactic and aggressive therapy with magnesium (10).

Some of neuroprotective properties of statins may play a role in cerebral VS and DCI prevention due to the ability to improve endothelial vasomotor function, increase endothelial cell fibrinolytic activity, reduce thrombogenic potential, inhibition of platelet activation, suppression of cytokine response during cerebral ischemia and improve cerebral blood flow (32). Results of statin therapy are controversial. A multicentre randomized phase III trial STASH, including 803 patients, not detected any benefit in the use of simvastatin for long-term or short-term outcome in patients with SAH (32). In contrast, meta-analysis of 13 studies show that statins significantly reduced the incidence of cerebral VS, DCI, neurologic deficit, and mortality, suggesting its efficacy (33). The latest AHA/ASA recommendations state that it is reasonable to administer statins to patients after SAH to prevent cerebral VS despite the lack of strong evidence of benefit (26). Therapy should be started within 48 hours after ictus and continued for 14 to 21 day (10). Apart from Nimodipine and statins, hemodynamic augmentation to improve cerebral perfusion is

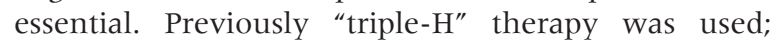
however, studies failed to show the benefit and the "triple- $\mathrm{H}$ " therapy is not recommended any longer $(10,29)$. New directions are to maintain euvolemia and induced hypertension. Rajajee at al. recommend to estimate the existence of hypoperfusion using the CT-perfusion imaging, jugular-bulb oximetry, microdialysis, brain-tissue oximetry and invasive cerebral blood flow monitoring and continuously monitor the response to treatment. Also, cardiac output should be monitored during inotropic therapy. PiCCO-directed hemodynamic therapy may improve outcomes in patients with poor-grade SAH. A more widely available point-of-care ultrasound can be used to determine fluid responsiveness in SAH, detect stressinduced cardiomyopathy and measure cardiac output. Daily lung ultrasound can detect incipient pulmonary edema ahead of clinical symptoms. The applications of those bedside tools have the potential to improve the efficacy of hemodynamic therapy and decrease complications (29).

Finally, in parallel to pharmacological interventions it is also important to prevent occurrence of anaemia with haemoglobin level $<9$ g/dL, hyperthermia, hyperglycaemia, hyponatremia and to optimize oxygenation, because they can contribute and worsen cerebral VS and DCI.

Invasive interventions. Blood products and fibrinolytics within the subarachnoid space and cisterns are associated with development of cerebral VS and DCI. Controversial data exists regarding the cleaning 
of subarachnoid space of blood by lumbar drainage of cerebrospinal fluid. A prospective, randomized, controlled trial LUMAS has shown that lumbar drainage of cerebrospinal fluid after aneurysmal $\mathrm{SAH}$ reduces the prevalence of delayed ischemic neurological deficit and improves early clinical outcome but fails to improve outcome after 6 months of ictus (30). Meta-analysis of 11 studies indicates that cerebrospinal fluid drainage reduced angiographic vasospasm, symptomatic vasospasm and delayed ischemic neurological deficit and reveals that lumbar drainage has lower complications, but more studies are required to verify and consolidate this conclusion (31). Cerebral angioplasty might be used in patients with symptomatic cerebral VS not responding to other medical measures but is not recommended for prophylaxis (Class IIa; Level of Evidence B). Also, selective intra-arterial vasodilator therapy is widely used in patients with symptomatic cerebral VS especially involving small distal vessels (Class IIa; Level of Evidence B).

Many other therapeutic interferences like use of endothelin receptor antagonists, tirilazad, fasulid, cilastazol, eicosapentaenoic acid, erythropoietin, corticosteroids, minocycline, deferoxamine, intrathecal thrombolytics, intra-arterial papaverine and others are not commonly used in practice (10).

\section{CONCLUSIONS}

$\mathrm{SAH}$ remains an unpredictable and invincible pathology. SAH is still associated with poor clinical outcome due to the development of early and late complications, and most survivors have long-term disability or cognitive impairment. The highest risk patients are those with initially low Glasgow coma scale and high grades of Fisher scale. A timely performed obliteration method either surgical or endovascular of the ruptured aneurysm is crucial and the only widely used standard treatment to prevent cerebral VS and DCI is Nimodipine.

\section{Conflict of interest: None}

Table 1. The Hunt and Hess Scale and survival rates (reprinted Marcolini 2019 (6))

\begin{tabular}{|l|l|c|}
\hline Grade & Criteria & Survival \\
\hline I & $\begin{array}{l}\text { Asymptomatic or mild headache } \\
\text { with slight nuchal rigidity }\end{array}$ & $70 \%$ \\
\hline II & $\begin{array}{l}\text { Moderate to severe headache, } \\
\text { nuchal rigidity, no neurological } \\
\text { deficit other than cranial nerve } \\
\text { palsy }\end{array}$ & $60 \%$ \\
\hline III & $\begin{array}{l}\text { Drowsiness, confusion, or mild } \\
\text { focal deficit }\end{array}$ & $50 \%$ \\
\hline IV & $\begin{array}{l}\text { Stupor, moderate to severe } \\
\text { hemiparesis, possibly early } \\
\text { decerebrate rigidity or vegetative } \\
\text { disturbance }\end{array}$ & $20 \%$ \\
\hline V & $\begin{array}{l}\text { Deep coma, decerebrate rigidity, } \\
\text { moribund appearance }\end{array}$ & $10 \%$ \\
\hline
\end{tabular}

Table 2. Prediction of cerebral vasospasm using the Fisher scale (Reprinted from https:// radiopaedia.org/articles/fisher-scale)

\begin{tabular}{|l|l|c|}
\hline Grade & Radiological finding & $\begin{array}{l}\text { The risk of } \\
\text { symptomatic } \\
\text { cerebral } \text { VS }\end{array}$ \\
\hline Fisher I & No SAH or IVH detected & $21 \%$ \\
\hline Fisher II & $\begin{array}{l}\text { Diffuse thin (<1 mm) SAH, no } \\
\text { clots }\end{array}$ & $25 \%$ \\
\hline Fisher III & $\begin{array}{l}\text { Localized clots and/or layers } \\
\text { of blood > mm in thickness, } \\
\text { no IVH }\end{array}$ & $37 \%$ \\
\hline Fisher IV & $\begin{array}{l}\text { Diffuse or no SAH, ICH or IVH } \\
\text { present }\end{array}$ & $31 \%$ \\
\hline
\end{tabular}

Abbreviations: VS - vasospasm; SAH - subarachnoid haemorrhage, IVH - intraventricular haemorrhage, $\mathrm{mm}$ - millimetres, ICH - intracerebral haemorrhage.

Table 3. SAH complications (reprinted from Daou 2019 (10))

\begin{tabular}{|l|l|l|}
\hline Early & Late & Chronic \\
\hline Rebleeding & $\begin{array}{l}\text { Cerebral } \\
\text { vasospasm }\end{array}$ & $\begin{array}{l}\text { Chronic } \\
\text { hydrocephalus } \\
\text { that may require } \\
\text { placement of a } \\
\text { shunt }\end{array}$ \\
\hline $\begin{array}{l}\text { Acute } \\
\text { hydrocephalus }\end{array}$ & $\begin{array}{l}\text { Delayed } \\
\text { cerebral } \\
\text { ischemia }\end{array}$ & $\begin{array}{l}\text { Behaviour, } \\
\text { personality and } \\
\text { memory changes }\end{array}$ \\
\hline Seizures & $\begin{array}{l}\text { Pituitary } \\
\text { dysfunction }\end{array}$ & $\begin{array}{l}\text { Need for further } \\
\text { retreatment for } \\
\text { recurrent or } \\
\text { residual aneurysm }\end{array}$ \\
\hline $\begin{array}{l}\text { Cardio- } \\
\text { pulmonary } \\
\text { issues and } \\
\text { stress-induced } \\
\text { cardiomyopathy }\end{array}$ & $\begin{array}{l}\text { Infection related } \\
\text { to surgery or } \\
\text { ventriculostomy }\end{array}$ & \\
\hline $\begin{array}{l}\text { Sodium and } \\
\text { electrolyte } \\
\text { abnormalities }\end{array}$ & \multicolumn{1}{|l}{} \\
\hline
\end{tabular}


Table 4. A comparison of surgical vs endovascular treatment

\begin{tabular}{|l|c|c|}
\hline & Surgical clipping & Endovascular treatment \\
\hline $\begin{array}{l}\text { Death and disability during the first } \\
\text { year, \% }\end{array}$ & 31 & 24 \\
\hline Death rate during 5-year follow-up, \% & 14 & 8 \\
\hline Technical complications, \% & 19 & Lhorter \\
\hline Time to secure aneurysm & Longer \\
\hline $\begin{array}{l}\text { Risk of epilepsy and significant } \\
\text { cognitive decline }\end{array}$ & Higher & 2.9 \\
\hline Incidence of late rebleeding, \% & 0.9 & 58 \\
\hline $\begin{array}{l}\text { Completely obliterated aneurysm, \% } \\
\text { of cases }\end{array}$ & 81 & $\begin{array}{c}\text { Anterior circulation aneurysms } \\
\text { Wide-necked aneurysms } \\
\text { Giant aneurysms }\end{array}$ \\
\hline $\begin{array}{l}\text { Recommended for treatment } \\
\text { Intraparenchymal hematoma }>50 ~ m l\end{array}$ & $\begin{array}{c}\text { Posterior circulation aneurysms } \\
\text { Poor grade SAH } \\
\text { Age }>70 \text { years }\end{array}$ \\
\hline
\end{tabular}

Abbreviations: \% - percent; ml - millilitres, SAH - subarachnoid haemorrhage
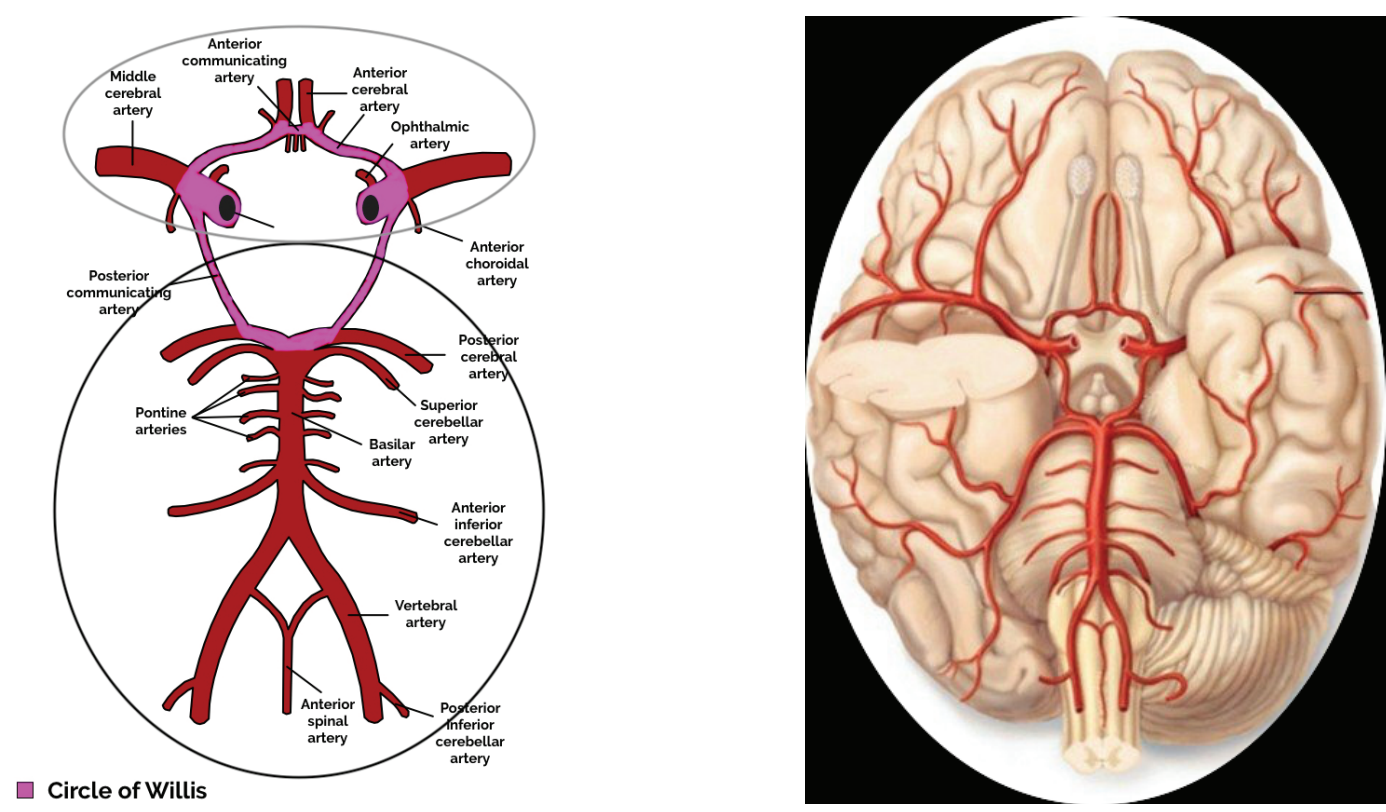

Fig. IA and 1B. The circle of Willis. Grey circulus - anterior circulation of brain; black circulus - posterior circulation of brain. Obtained picture 1 A from https://geekymedics.com/wp-content/uploads/2018/09/ Circle_of_Willis_en.jpg and IB from https://www.scienceabc.com/humans/circle-of-willis-anatomydiagram-and-functions.html 


\section{REFERENCES}

1. Etminan N., Chang HS, Hackenberg K, de Rooij NK, Vergouwen MDI, Rinkel GJE. Algra A. Worldwide Incidence of Aneurysmal Subarachnoid Hemorrhage According to Region, Time Period, Blood Pressure, and Smoking Prevalence in the Population: A Systematic Review and Meta-analysis. JAMA Neurol. 2019; 76(5): 588-597.

2. D'Souza, S. Aneurysmal Subarachnoid Hemorrhage. J Neurosurg Anesthesiol 2015; 27(3): 222240.

3. Suarez JI, Tarr RW, Selman WR. Aneurysmal subarachnoid hemorrhage. N Engl J Med. 2006; 354:387-396.

4. Athanasios K. Petridis, Marcel A. Kamp, Jan F. Cornelius, Thomas Beez, Kerim Baseoglu, Bernd Turowski, and Hans-Jakob Steiger. Aneurysmal Subarachnoid Hemorrhage. Dtsch Arztebl Int 2017; 114: 226-36.

5. Westerlaan HE, van Dijk JM, Jansen-van der Weide MC, de Groot JC, Groen RJ, Mooij JJ, Oudkerk M. Intracranial aneurysms in patients with subarachnoid hemorrhage: CT angiography as a primary examination tool for diagnosis - systematic review and meta-analysis. Radiology. 201 1; 258:134-145

6. Evie Marcolini, MD and Jason Hine, MD. Approach to the Diagnosis and Management of Subarachnoid Hemorrhage. Western Journal of Emergency Medicine. March 2019; Volume 20, No. 2: 203-211.

7. Rosen DS, Macdonald RL. Subarachnoid hemorrhage grading scales: a systematic review. Neurocrit Care. 2005; 2:110-118.

8. Liu, Z., Ajimu K, Yalikun N, Zheng Y, Xu F. Potential Therapeutic Strategies for Intracranial Aneurysms Targeting Aneurysm Pathogenesis. Front Neurosci. 2019; 13:1238.

9. Helene Hurth, Ulrich Birkenhauer, Jochen Steiner, Dennis Schlak, Florian Hennersdorf, Florian H. Ebner. Delayed Cerebral Ischemia in Patients with Aneurysmal Subarachnoid Hemorrhage - Serum D-dimer and C-reactive Protein as Early Markers. J Stroke Cerebrovasc Dis. 2019; 104558.

10. Badih J. Daou, Sravanthi Koduri, B. Gregory Thompson, Neeraj Chaudhary, and Aditya S. Pandey. Clinical and experimental aspects of aneurysmal subarachnoid hemorrhage. CNS Neurosci Ther. 2019 Oct; 25(10): 1096-1112.

11. Constantine Constantoyannis, George C. Sakellaropoulos, George C. Kagadis, Paraskevi F. Katsakiori, Theodore Maraziotis, George C. Nikiforidis, Nikolas Papadakis. Transcranial cerebral oximetry and transcranial doppler sonography in patients with ruptured cerebral aneurysms and delayed cerebral vasospasm. Med Sci Monit. 2007; 13(10): MT35-40.

12. Varinder S. Alg, BSc, Reecha Sofat, MSc, Henry Houlden, PhD, and David J. Werring, PhD. Genetic risk factors for intracranial aneurysms. Neurology. 2013 Jun 4: 80(23): 2154-2165.
13. Trine H. Andreasen, Jiri Bartek Jr, Morten Andresen, Jacob B. Springborg, and Bertil Romner. Modifiable Risk Factors for Aneurysmal Subarachnoid Hemorrhage. Stroke. 5 Nov 2013; 44:3607-3612.

14. Xi-Yang Yao, Cai-Qi Jiang, Gen-Lai Jia, and Gang Chen. Diabetes mellitus and the risk of aneurysmal subarachnoid haemorrhage: A systematic review and meta-analysis of current evidence. J Int Med Res. 2016 Dec; 44(6): 1141-1155.

15. Walter N. Kernan, M.D., Catherine M. Viscoli, Ph.D., Lawrence M. Brass, M.D., Joseph P. Broderick, M.D., Thomas Brott, M.D., Edward Feldmann, M.D., Lewis B. Morgenstern, M.D., Janet Lee Wilterdink, M.D., and Ralph I. Horwitz, M.D. Phenylpropanolamine and the Risk of Hemorrhagic Stroke. N Engl J Med. December 21, 2000; 343:1826-1832.

16. Sureerat Suwatcharangkoon, MD; Emma Meyers, BA; Cristina Falo, PhD; J. Michael Schmidt, PhD; Sachin Agarwal, MD, MPH; Jan Claassen, MD, PhD; Stephan A. Mayer, MD. Loss of Consciousness at Onset of Subarachnoid Hemorrhage as an Important Marker of Early Brain Injury. JAMA Neurol. 2016; 73(1):28-35.

17. Sheng Chen, Qian Li, Haijian Wu, Paul R. Krafft, Zhen Wang, and John H. Zhang. The Harmful Effects of Subarachnoid Hemorrhage on Extracerebral Organs. Biomed Res Int. 2014; 858496.

18. Lieveke A. R. Degen, Sanne M. Dorhont Mees, Ale Algra and Gariäl J. E. Rinkel. Interobserver Variability of Garding Scales for Aneurysmal Subarachnoid Hemorrhage. Stroke. 2011; 42: 15461549.

19. Brit Long MD, Alex Koyfman MD. Controversies in the Diagnosis of Subarachnoid Hemorrhage. J Emerg Med. 2016 Jun; 50(6): 839-47.

20. Chao Tang, Tian-Song Zhang, Liang-Fu Zhou. Risk Factors for Rebleeding of Aneurysmal Subarachnoid Hemorrhage: A Meta-Analysis. PLoS One. 2014; 9(6): e99536.

21. Chen S, Luo J, Reis C, Manaenko A, Zhang J. Hydrocephalus after Subarachnoid Hemorrhage:Pathophysiology, Diagnosis, and Treatment. Biomed Res Int. 2017; 8584753.

22. Barret Rush, Katie Wiskar, Clark Fruhstorfer, Paul Hertz. Association between seizures and mortality in patients with aneurysmal subarachnoid hemorrhage: A nationwide retrospective cohort analysis. Seizure: Eur J Epilepsy. 2016; 41: 66-69.

23. Charles L. Francoeur and Stephan A. Mayer. Management of delayed cerebral ischemia after subarachnoid hemorrhage. Crit Care. 2016; 20: 277.

24. J. Max Findlay, Joshua Nisar, Tim Darsaut. Cerebral Vasospasm: A Review. Can J Neurol Sci. 2016; 43: $15-32$.

25. Limbruno U, De Caterina R. Vasomotor effects of iodinated contrast media: just side effects? Curr Vasc Pharmacol. 2003; 1(3): 321-8. 
26. E. Sander Connolly, Jr, MD, FAHA, Chair; Alejandro A. Rabinstein, MD, Vice Chair; J. Ricardo Carhuapoma, MD, FAHA; Colin P. Derdeyn, MD, FAHA; Jacques Dion, MD, FRCPC; Randall T. Higashida, MD, FAHA; Brian L. Hoh, MD, FAHA; Catherine J. Kirkness, PhD, RN; Andrew M. Naidech, MD, MSPH; Christopher S. Ogilvy, MD; Aman B. Patel, MD; B. Gregory Thompson, MD; Paul Vespa, MD, FAAN; on behalf of the American Heart Association Stroke Council, Council on Cardiovascular Radiology and Intervention, Council on Cardiovascular Nursing, Council on Cardiovascular Surgery and Anesthesia, and Council on Clinical Cardiology. Guidelines for the Management of Aneurysmal Subarachnoid Hemorrhage. A Guideline for Healthcare Professionals from the American Heart Association/American Stroke Association. Stroke. 2012; 43:1711-1737.

27. Tomoya Okazaki and Yasuhiro Kuroda. Aneurysmal subarachnoid hemorrhage: intensive care for improving neurological outcome. Journal of Intensive Care. 2018; 6:28.

28. Sanne M Dorhout Mees, MD, Prof Ale Algra, MD, Prof W Peter Vandertop, MD, Pop van Kooten, MD, Hans AJM Kuijsten, MD, Jelis Boiten, MD, Prof Robert J van Oostenbrugge, MD, Rustam Al-Shahi Salman, PhD, Pablo M Lavadas, MD, Prof Gabriel JE Rinkel, MD, Walter M van den Bergh, $\mathrm{MD}$ on behalf of the MASH-2 study group. Magnesium for aneurysmal subarachnoid haemorrhage (MASH-2): a randomized placebo-controlled trial. The Lancet. July 07, 2012; 38(9836): 44-49.

29. Venkatakrishna Rajajee, Aditya S. Pandey, Craig A. Williamson. Subarachnoid Hemorrhage and Therapy Formerly Known as "Triple-H"-New Directions. World Neurosurg. 2019 Jul; 127:500-501.

30. Yahia Z. Al-Tamimi, MRCS; Deepti Bhargava, MRCS; Richard G. Feltbower, PhD; Gregory Hall, PhD; Anthony J.P. Goddard, FRCR; Audrey C. Quinn, FFARCSI; Stuart A. Ross, FRCS(SN). Lumbar Drainage of Cerebrospinal Fluid After Aneurysmal Subarachnoid Hemorrhage. A Prospective, Randomized, Controlled Trial (LUMAS). Stroke. 2012; 43:677-682.
31. Cong Qian, MD, Xiaobo Yu, MD, Jingyin Chen, $\mathrm{MD}$, Chi Gu, MD, Lin Wang, MD, Gao Chen, MD, and Yuying Dai, MD. Effect of the drainage of cerebrospinal fluid in patients with aneurismal subarachnoid hemorrhage. A meta-analysis. Medicine (Baltimore). 2016 Oct; 95(41): e5140.

32. Peter J Kirkpatrick, Carole L Turner, Christopher Smith, Peter J Hutchinson, Gordon D Murray, for the STASH Collaborators. Simvastatin in aneurysmal subarachnoid haemorrhage (STASH): a multicentre randomised phase 3 trial. Lancet Neurol 2014; 13: 666-75.

33. Jianguo Shen, Jian Shen, Kuncan Zhu, Haihang Zhou, Heping Tian, Gongjie Yu. Efficacy of Statins in Cerebral Vasospasm, Mortality, and Delayed Cerebral Ischemia in Patients with Aneurysmal Subarachnoid Hemorrhage: A Systematic Review and Meta-Analysis of Randomized Controlled Trials. World Neurosurgery. November 2019; 131: e65-e73.

34. Simonassi, Francesca MD; Ball, Lorenzo MD; Badenes, Rafael PhD; Millone, Marco MD; Citerio Giuseppe MD; Zona, Gianluigi PhD; Pelosi, Paolo MD; Robba, Chiara PhD. Hemodynamic Monitoring in Patients with Subarachnoid Hemorrhage. A Systematic Review and Meta-Analysis. Neurosurg Anesthesiol. 2020 Jan 31 - Publish Ahead of Print.

\section{Address:}

Ieva Buce-Satoba

Clinics of Anesthesiology and Intensive Care

Riga East University Hospital

Hipokrata iela 2

Riga, Latvia, LV-1038

Email: ieva.buce_satoba@icloud.com 